\title{
THE LEVEL SETS OF ITERATED BROWNIAN MOTION
}

\author{
BY \\ KrzyszTof BurdzY ${ }^{1}$ AND DAVAR KHOSHNEVISAN \\ University of Washington $\mathcal{G}$ University of Utah
}

Abstract. We show that the Hausdorff dimension of every level set of iterated Brownian motion is equal to $3 / 4$.

$\S 1$. Introduction and Main Result. Suppose $(\Omega, \mathcal{F}, \mathbf{P})$ is a probability space, rich enough to carry three independent Brownian motions, $X^{+}, X^{-}$and $Y$, all starting from the origin. Iterated Brownian motion (IBM) is the process defined by $Z(t)=X(Y(t))$, where $X(t)=X^{+}(t) 1_{[0, \infty)}(t)+X^{-}(-t) 1_{(-\infty, 0)}(t)$. The probabilistic and analytical properties of IBM and related processes have been the subject of recent vigorous investigations; see Bertoin [B], Burdzy [B1,B2], Csáki et al. [CsCsFR1,CsCsFR2], Deheuvels and Mason [DM], Funaki [F], Hu et al. [HPS], Hu and Shi [HS], Khoshnevisan and LEWIS [KL1,KL2] and SHI [S] together with their combined references. Define the setvalued $x$-level set process, $\mathcal{L}_{x}(t)$, by

$$
\mathcal{L}_{x}(t)=\{0 \leq s \leq t: Z(s)=x\}, \quad \text { for all } x \in \mathbf{R}^{1} .
$$

The main result of this paper is the following analogue of Paul Lévy's well-known result for Brownian motion (see Itô AND MCKeAn [IM] and AdLer [A]):

(1.2) Theorem. Let $\operatorname{dim}_{H}$ denote Hausdorff dimension. Then, outside a single null set,

$$
\operatorname{dim}_{H}\left(\mathcal{L}_{x}(t)\right)=\frac{3}{4},
$$

simultaneously for all $t \geq 0$ and all $x$ in the interior of $Z([0, t])$.

Here and throughout, if $f: \mathbf{R}^{1} \mapsto \mathbf{R}^{1}$ is Borel measurable and $A \subset \mathbf{R}^{1}$ is measurable, then $f(A)=\{y: y=f(x)$ for some $x \in A\}$.

1 Research supported in part by NSF grant DMS 91-00244 and AMS Centennial Research Fellowship 
The proof of Theorem (1.2) uses a capacity argument due to Frostman (see AdLER [A]) and relies on the following which has been discovered independently and at the same time by CsÁki ET AL. [CsCsFR2]:

(1.3) Proposition. There exists an almost surely jointly continuous family of "local times", $\left\{\ell_{t}^{a} ; t \geq 0, a \in \mathbf{R}^{1}\right\}$, such that for all Borel measurable integrable functions, $f$ : $\mathbf{R}^{1} \mapsto \mathbf{R}^{1}$ and all $t \geq 0$,

$$
\int_{0}^{t} f(Z(s)) d s=\int_{-\infty}^{\infty} f(a) \ell_{t}^{a} d a
$$

Acknowledgements. We wish to thank T.M. Lewis for many enjoyable discussions. Also many thanks are due to J. Bertoin as well as E. Csáki and M. Csörgő for sending us the articles, [B] and [CsCsFR1,CsCsFR2].

$\S 2$. Local Times. If $B$ is any Brownian motion, its process of local times will be denoted by $L_{t}^{a}(B)$. These satisfy the following occupation density formula: for any Borel measurable $f: \mathbf{R}^{1} \mapsto \mathbf{R}^{1}$ and all $t \geq 0$,

$$
\int_{0}^{t} f(B(r)) d r=\int_{-\infty}^{\infty} f(a) L_{t}^{a}(B) d a
$$

For a stochastic calculus description as well as many deep properties of local times see Revuz AND YoR [RY]. Proposition (1.3) is a consequence of the following real variable fact:

(2.2) Proposition. Let $K(b, d a)$ be the kernel defined by

$$
K(b, d a)=L_{d a}^{b}\left(X^{+}\right)+L_{d a}^{b}\left(X^{-}\right)
$$

Then the local times $\ell$ are given by

$$
\ell_{t}^{b}=\int_{Y([0, t])} L_{t}^{a}(Y) K(b, d a)
$$

Proof. Let $f: \mathbf{R}^{1} \mapsto \mathbf{R}^{1}$ be as in Proposition (1.3). Viewing $f(Z)$ as $(f \circ X)(Y)$, we see from (2.1) that for all $t \geq 0$, a.s.,

$$
\begin{aligned}
\int_{0}^{t} f(Z(s)) d s & =\int_{-\infty}^{\infty}(f \circ X)(a) L_{t}^{a}(Y) d a \\
& =\int_{0}^{\infty}\left(f \circ X^{+}\right)(a) L_{t}^{a}(Y) d a+\int_{-\infty}^{0}\left(f \circ X^{-}\right)(a) L_{t}^{a}(Y) d a
\end{aligned}
$$


By (2.1) and a monotone class argument, for any jointly measurable $F: \mathbf{R}^{1} \times \mathbf{R}_{+}^{1} \mapsto \mathbf{R}^{1}$,

$$
\int_{0}^{\infty} F\left(X^{ \pm}(s), s\right) d s=\int_{-\infty}^{\infty} \int_{0}^{\infty} F(a, t) L_{d t}^{a}\left(X^{ \pm}\right) d a .
$$

Applying (2.3),

$$
\begin{aligned}
\int_{0}^{t} f(Z(s)) d s=\int_{-\infty}^{\infty} f & (b) d b \int_{0}^{\infty} L_{t}^{a}(Y) L_{d a}^{b}\left(X^{+}\right) \\
& +\int_{-\infty}^{\infty} f(b) d b \int_{0}^{\infty} L_{t}^{-a}(Y) L_{d a}^{b}\left(X^{-}\right)
\end{aligned}
$$

Since $a \mapsto L_{t}^{a}(Y)$ is a.s. supported on $Y([0, t])$,

$$
\begin{aligned}
\int_{0}^{t} f(Z(s)) d s= & \int_{-\infty}^{\infty} f(b) d b \int_{\mathbf{R}_{+}^{1} \cap Y([0, t])} L_{t}^{a}(Y) L_{d a}^{b}\left(X^{+}\right) \\
& +\int_{-\infty}^{\infty} f(b) d b \int_{\mathbf{R}_{+}^{1} \cap Y([0, t])} L_{t}^{a}(Y) L_{d a}^{b}\left(X^{-}\right) .
\end{aligned}
$$

The proposition follows from a change of variables. The joint continuity of $\ell_{t}^{a}$ follows from that of $L_{t}^{a}(B)$ for any Brownian motion, $B$; see REvuz AND Yor [RY].

(2.4) Proposition. For any $T>0$, almost surely,

$$
\limsup _{\varepsilon \rightarrow 0} \sup _{a \in \mathbf{R}^{1}} \sup _{0 \leq t \leq T} \frac{\ell_{t+\varepsilon}^{a}-\ell_{t}^{a}}{\varepsilon^{3 / 4}(\ln (1 / \varepsilon))^{5 / 4}} \leq 2^{3 / 2}, \quad \text { a.s. . }
$$

With more work, one can improve the upper bound of $2^{3 / 2}$ in the above. However, we do not even know whether the power of the logarithm is the correct one. Therefore, we will be satisfied with our simple proof of the upper bound.

Proof. By Proposition (2.2),

$$
\begin{aligned}
\ell_{t+\varepsilon}^{a}-\ell_{t}^{a}= & \int_{Y([0, t])}\left(L_{t+\varepsilon}^{r}(Y)-L_{t}^{r}(Y)\right) K(a, d r) \\
& \quad+\int_{Y([0, t+\varepsilon]) \backslash Y([0, t])} L_{t+\varepsilon}^{r}(Y) K(a, d r) \\
= & \mathrm{I}_{(2.5)}+\Pi_{(2.5)} .
\end{aligned}
$$

For all $r \in Y([0, t]) \backslash Y([t, t+\varepsilon]), L_{t+\varepsilon}^{r}(Y)=L_{t}^{r}(Y)$. Hence, as $\varepsilon \rightarrow 0^{+}$,

$$
\begin{aligned}
\mathrm{I}_{(2.5)} & =\int_{Y([t, t+\varepsilon]) \cap Y([0, t])}\left(L_{t+\varepsilon}^{r}(Y)-L_{t}^{r}(Y)\right) K(a, d r) \\
& \leq \sup _{r}\left(L_{t+\varepsilon}^{r}(Y)-L_{t}^{r}(Y)\right) K(a, Y([t, t+\varepsilon])) \\
& \leq(1+o(1)) \sqrt{2 \varepsilon \ln (1 / \varepsilon)} K(a, Y([t, t+\varepsilon])),
\end{aligned}
$$


uniformly over all $a \in \mathbf{R}^{1}$ and all $0 \leq t \leq T$. We have used the uniform modulus of continuity of local times in time; see Lemma 5(c) of PERKINs $[\mathrm{P}]$. By Lévy's modulus of continuity $([\mathrm{RY}])$, as $\varepsilon \rightarrow 0^{+}$,

$$
|Y([t, t+\varepsilon])| \leq(1+o(1)) \sqrt{2 \varepsilon \ln (1 / \varepsilon)}
$$

uniformly over all $0 \leq t \leq T$. Hence, by MCKEAN [Mc] and the independence of $X^{+}$and $X^{-}$, as $\varepsilon \rightarrow 0^{+}$, uniformly over all $a \in \mathbf{R}^{1}$ and $0 \leq t \leq T$,

$$
\begin{aligned}
K(a, Y([t, t+\varepsilon])) & \leq(1+o(1)) \sqrt{2 \sqrt{2 \varepsilon \ln (1 / \varepsilon)} \mid \ln \sqrt{2 \varepsilon \ln (1 / \varepsilon)}} \\
& =(1+o(1)) 2^{1 / 4} \varepsilon^{1 / 4}(\ln (1 / \varepsilon))^{3 / 4} .
\end{aligned}
$$

This implies that as $\varepsilon \rightarrow 0^{+}$,

$$
\mathrm{I}_{(2.5)} \leq(1+o(1)) 2^{3 / 4} \varepsilon^{3 / 4}(\ln (1 / \varepsilon))^{5 / 4} .
$$

The bound for $\Pi_{(2.5)}$ is very similar. Note that for all $r \in Y([0, t+\varepsilon]) \backslash Y([0, t]), L_{t}^{r}(Y)=0$. Hence, making similar arguments as above, we see that as $\varepsilon \rightarrow 0^{+}$, uniformly over all $a \in \mathbf{R}^{1}$ and $0 \leq t \leq T$,

$$
\begin{aligned}
\Pi_{(2.5)} & =\int_{Y([0, t+\varepsilon]) \backslash Y([0, t])}\left(L_{t+\varepsilon}^{r}(Y)-L_{t}^{r}(Y)\right) K(a, d r) \\
& \leq \sup _{r}\left(L_{t+\varepsilon}^{r}(Y)-L_{t}^{r}(Y)\right) K(a, Y([t, t+\varepsilon])) \\
& \leq(1+o(1)) \sqrt{2 \varepsilon \ln (1 / \varepsilon)} \sqrt{2|Y([t, t+\varepsilon])| \cdot|\ln | Y([t, t+\varepsilon])||} \\
& =(1+o(1)) 2^{3 / 4} \varepsilon^{3 / 4}(\ln (1 / \varepsilon))^{5 / 4} .
\end{aligned}
$$

Together with (2.5) and (2.6), the above implies the result.

$\S 3$. The proof of Theorem (1.2). Once there is a modulus of continuity of local times (in $t$ ), we proceed by Frostman's capacity method as outlined in ADLER [A], for example. Recall from KHOSHNEvisAn AND Lewis [KL] that for any $T>0$, almost surely,

$$
\lim _{\varepsilon \rightarrow 0} \sup _{0 \leq t \leq T} \frac{|Z(t+\varepsilon)-Z(t)|}{\varepsilon^{1 / 4}(\ln (1 / \varepsilon))^{3 / 4}}=1, \quad \text { a.s. . }
$$

In particular, we see that $Z$ is Hölder continuous of order $\gamma<1 / 4$. By Proposition (1.3) and Lemma 7 of $[\mathrm{A}]$, simultaneously over all $x \in \mathbf{R}^{1} \operatorname{dim}_{H} \mathcal{L}_{x}(t) \leq 3 / 4$. Moreover, by Proposition (2.4), $t \mapsto \ell_{t}^{a}$ is Hölder continuous of order $\gamma<3 / 4$, uniformly in $a \in \mathbf{R}^{1}$. By Frostman's lemma, (see the proof of Lemma 6 of $[\mathrm{A}]$ ), simultaneously over all $x$ in the interior of $Z([0, t]), \operatorname{dim}_{H} \mathcal{L}_{x}(t) \geq 3 / 4$. This proves the result. 
We conclude this section with some open problem.

Problem 1. Define $Z^{+}(t)=\sup _{0 \leq s \leq t} Z(s)$. BerToIn [B] proves that for all $T>0$, almost surely,

$$
\lim _{\varepsilon \rightarrow 0} \sup _{0 \leq t \leq T} \frac{\left|Z^{+}(t+\varepsilon)-Z^{+}(t)\right|}{\varepsilon^{1 / 4}(\ln (1 / \varepsilon))^{3 / 4}}=\frac{1}{2^{1 / 4} \cdot 3^{3 / 4}} .
$$

In light of (3.1), this says that $Z^{+}$is smoother than $Z$. Is there a probabilistic explanation for this, in terms of (say) path decompositions?

Problem 2. Define

$$
\mathcal{S}^{+}(t) \triangleq\{t \in[0,1]: Z(t)>Z(s) \text { for all } s<t\}
$$

According to Bertoin [B], the Hausdorff dimension of $\mathcal{S}^{+}(t)$ is almost surely $1 / 4$. This is in sharp contrast with Theorem (1.2) and the analogous result for Brownian motion which is a consequence of Lévy's characterization of Brownian motion. Is there a probabilistic explanation for this apparent difference?

Problem 3. By being more careful, it is possible to show that $\varphi$-Hausdorff measure of $\mathcal{L}_{0}(t)$ is a.s. (strictly) positive, if $\varphi(\varepsilon)=\varepsilon^{3 / 4}(\ln (1 / \varepsilon))^{5 / 4}$. Is this sharp? For the corresponding problem for $\mathcal{S}^{+}(t)$, see Bertoin [B].

\section{References.}

[A] R.J. AdLER (1978). The uniform dimension of the level sets of a Brownian sheet, Ann. Prob. 6 509-515.

[B] J. Bertoin (1995). Iterated Brownian motion and Stable (1/4) subordinator, to appear in Prob. and Stat. Lett.

[B1] K. Burdzy (1993). Some path properties of iterated Brownian motion. Sem. Stoch. Proc. 1992, 67-87 (Ed. K.L. Chung, E. Çinlar and M.J. Sharpe) Birkhäuser, Boston.

[B2] K. Burdzy (1994). Variation of iterated Brownian motion. Measure-valued Processes, Stochastic Partial Differential Equations and Interacting Systems, (Ed. D.A. Dawson) CRM Proceedings and Lecture Notes, 5 35-53.

[CsCsFr1] E. Csáki, M. Csörgö, A. Földes And P. RÉvész (1995). Global Strassen type theorems for iterated Brownian motion, to appear in Stoch. Proc. Their Appl.

[CsCsFr2] E. Csáki, M. Csörgö, A. Földes and P. RÉvész (1995). The local time of iterated Brownian motion, Preprint. 
[DM] P. Deheuvels and D.M. Mason (1992). A functional LIL approach to pointwise Bahadur-Kiefer theorems, Prob. in Banach Spaces, 8, 255-266 (eds.: R.M. Dudley, M.G. Hahn and J. Kuelbs)

[F] T. Funaki (1979). A probabilistic construction of the solution of some higher order parabolic differential equations, Proc. Japan Acad. 55, 176-179.

[HPS] Y. Hu, D. Pierre Lotti Viaud and Z. Shi (1994). Laws of the iterated logarithm for iterated Wiener processes, to appear in J. Theor. Prob.

[HS] Y. Hu AND Z. SHi (1994). The Csörgö-Révész modulus of non-differentiability of iterated Brownian motion, to appear in Stoch. Proc. Their Appl..

[IM] K. Itô And H.P. McKean (1965). Diffusion Processes and Their Sample Paths, Springer, Berlin, Heidelberg.

[KL1] D. Khoshnevisan And T.M. Lewis (1995). Chung's law of the iterated logarithm for iterated Brownian motion, to appear in Ann. Inst. Hen. Poinc.: Prob. et Stat.

[KL2] D. Khoshnevisan and T.M. Lewis (1995). The modulus of continuity for iterated Brownian motion, to appear in J. Theoretical Prob.

[Mc] H.P. McKean (1962). A Hölder condition for Brownian local time, J. Math. Kyoto Univ., 1-2, 195-201.

[P] E.A. Perkins (1981). The exact Hausdorff measure of the level sets of Brownian motion, Z. Wahr. verw. Geb. 58, 373-388.

[RY] D. Revuz And M. Yor (1991). Continuous Martingales and Brownian Motion, Springer, New York.

[S] Z. SHI (1994). Lower limits of iterated Wiener processes, to appear in Stat. Prob. Lett.

K. BURDZY

Department of Mathematics

University of Washington

Seattle, WA. 98195

burdzy@math.washington.edu
D. KHOSHNEVISAN

Department of Mathematics

University of Utah

Salt Lake City, UT. 84112

davar@math.utah.edu 